BMJ Surgery, Interventions, $\&$ Health Technologies

\section{Selecting esophageal cancer patients for lymphadenectomy after neoadjuvant chemoradiotherapy: a modeling study}

To cite: Scholte M, de Gouw DJJM, Klarenbeek BR, et al. Selecting esophageal cancer patients for lymphadenectomy after neoadjuvant chemoradiotherapy: a modeling study. BMJ Surg Interv Health Technologies 2020;2:e000027. doi:10.1136/ bmjsit-2019-000027

- Additional material is published online only. To view please visit the journal online (http://dx.doi.org/10.1136/ bmjsit-2019-000027).

MS and DJdG contributed equally.

Received 06 January 2020 Revised 03 April 2020 Accepted 09 April 2020

\section{Check for updates}

C Author(s) (or their employer(s)) 2020. Re-use permitted under CC BY-NC. No commercial re-use. See rights and permissions. Published by BMJ.

${ }^{1}$ Operating Rooms, Radboud Institute of Health Sciences, Radboudumc, Nijmegen, The Netherlands

${ }^{2}$ Surgery, Radboud Institute of Health Sciences, Radboudumc, Nijmegen, The Netherlands ${ }^{3}$ Operating Rooms and Health Evidence, Radboud Institute of Health Sciences, Radboudumc, Nijmegen, The Netherlands

Correspondence to

Ms Mirre Scholte;

mirre.scholte@radboudumc.nl

\section{ABSTRACT}

Objectives Two-thirds of patients do not harbor lymph node (LN) metastases after neoadjuvant chemoradiotherapy (nCRT). Our aim was to explore under which circumstances a selective lymph node dissection (LND) strategy, which selects patients for LND based on the restaging results after nCRT, has added value compared with standard LND in esophageal cancer. Design A decision tree with state-transition model was developed. Input data on short-term and long-term consequences were derived from literature. Sensitivity analyses were conducted to assess promising scenarios and uncertainty.

Setting Dutch healthcare system.

Participants Hypothetical cohort of esophageal cancer patients who have already received nCRT and are scheduled for esophagectomy.

Interventions A standard LND cohort was compared with a cohort of patients that received selective LND based on the restaging results after nCRT.

Main outcome measures Quality-adjusted life years (QALYS), residual LN metastases and LND-related complications.

Results Selective LND could have short-term benefits, that is, a decrease in the number of performed LNDs and LND-related complications. However, this may not outweigh a slight increase in residual LN metastases which negatively impacts QALYs in the long-term. To accomplish equal QALYs as with standard LND, a new surgical strategy should have the same or higher treatment success rate as standard LND, that is, should show equal or less recurrences due to residual LN metastases.

Conclusions The reduction in LND-related complications that is accomplished by selecting patients for LND based on restaging results after nCRT seems not to outweigh a QALY loss in the long-term due to residual LN metastases. Despite the short-term advantages of selective LND, this strategy can only match long-term QALYs of standard LND when its success rate equals the success rate of standard LND.

\section{INTRODUCTION}

Primary treatment of resectable esophageal cancer consists of neoadjuvant chemoradiotherapy (nCRT) followed by surgical resection of the esophagus including the regional

\section{Key messages}

What is already known about this subject?

- As many as $65 \%$ of esophageal cancer patients do not harbor lymphnode (LN) metastases after neoadjuvant chemoradiotherapy (nCRT), which challenges the need for lymphadenectomy in a substantial part of patients. Ideally, only patients who harbor $\mathrm{LN}$ metastases after nCRT would undergo lymphnode dissection (LND), but the added value of such a selective LND approach is currently unknown.

What are the new findings?

- Our modeling study shows that selecting patients for LND based on restaging results after nCRT could reduce LND-related complications, but this beneficial effect seems not to outweigh a loss in quality of life in the long-term due to a slight expected increase in patients with residual LN metastases.

\section{How might these results affect future} research or surgical practice?

- The consequences of having residual LN metastases are far more serious than those of short-term complications. Despite the short-term advantages of selective LND based on restaging results after $\mathrm{nCRT}$, this strategy can only match long-term QALYs of standard LND when its success rate equals the success rate of standard LND.

lymph nodes (LNs) ${ }^{1-3}$ However, only $31 \%$ to $38 \%$ of patients harbor LN metastases after nCRT. ${ }^{145}$ This implies that in up to $69 \%$ of patients the lymph node dissection (LND) and its associated morbidity is needless. If we would adopt a new surgical strategy in which the restaging results after nCRT are used to select patients suspicious for harboring locoregional LN metastases, part of surgeryrelated complications could possibly be avoided. ${ }^{6}$ Our hypothesis is that by reducing the number of unnecessary LNDs, unnecessary morbidity could be prevented.

Ideally, consequences (eg, therapeutic consequences, quality of life (QoL) and survival) and relevant uncertainties of new 
surgical strategies should be evaluated in an early stage of development, for example, in the pre- IDEAL stage (framework that describes the stages of innovation in surgery: Idea, Development, Exploration, Assessment, Long-term study). In that way, research and development of new surgical strategies can be steered in a way that they add most value to patients, healthcare and society. Therefore, we have previously conducted an expert case vignette survey in which we collected opinions from esophageal surgeons worldwide $(n=89)$ via case presentations. ${ }^{6}$ In this paper, we describe the results of a modeling study based on scientific literature and the results of the expert case vignette survey, in which we compare standard LND practice with a hypothetical new selective LND strategy in which the restaging results after nCRT are used to select patients for LND. The model analyses the short-term and long-term consequences of both strategies and explores promising scenarios.

\section{MATERIALS AND METHODS Model development}

We developed a model to evaluate complications, QoL and survival associated with standard LND for the diagnosis and treatment of LN metastases in patients with resectable distal esophageal cancer, and compared this to a hypothetical new strategy in which the restaging results after nCRT are used to select patients for LND. We assumed patients were 60 years old, the mean age at which esophageal cancer is diagnosed. ${ }^{1}$ Furthermore, we assumed all patients had already received nCRT, had no distant metastases and were eligible for both strategies. The model consisted of a decision tree to model the primary treatment, and a state-transition model for the long-term follow-up of patients (figures 1 and 2). Based on clinical guidelines and expert interviews, the model was designed to resemble the clinical situation. The model was validated in accordance with the AdViSHE checklist (Assessment of the Validation Status of HealthEconomic decision models), by means of consulting clinical experts, cross-validation with relevant literature, and extreme value and subunit testing. ${ }^{7}$ Consequences of the hypothetical new test strategy were based on a worldwide case vignette study among esophageal surgeons. ${ }^{6}$

\section{Decision tree}

For each strategy, patients could either have locoregional LN metastases or no locoregional LN metastases after nCRT. In the standard LND strategy, all patients received routine esophagectomy with LND. ${ }^{1}$ In the selective LND strategy, patients were selected for LND based on the restaging results after nCRT. Patients with low suspicion of LN metastases received esophagectomy without LND, while patients with high suspicion of LN metastases received esophagectomy with LND. As a result, four groups of patients can be distinguished: first, patients with low suspicion of LN metastases, in whom LN metastases were not present. Those patients benefited from the esophagectomy only. Second, patients with low suspicion of LN metastases, in whom LN metastases turned out to be present. Those patients were falsely treated with esophagectomy only and have residual LN metastases as a result. Third, patients with high suspicion of LN metastases, in whom LN metastases were indeed present. Those patients were successfully treated with esophagectomy and LND. Last, patients with high suspicion of LN metastases, in whom LN metastases were not present. Those patients received unnecessary LND. Furthermore, we assumed that in patients selected for LND who harbor LN metastases, these were always included in the LND.

In our model, we included complications that could potentially be avoided if LND is omitted. International experts of a previous case vignette survey determined that the number of pneumonias, chyle leakages and recurrent laryngeal nerve pareses would decrease when LND would be omitted. ${ }^{6}$
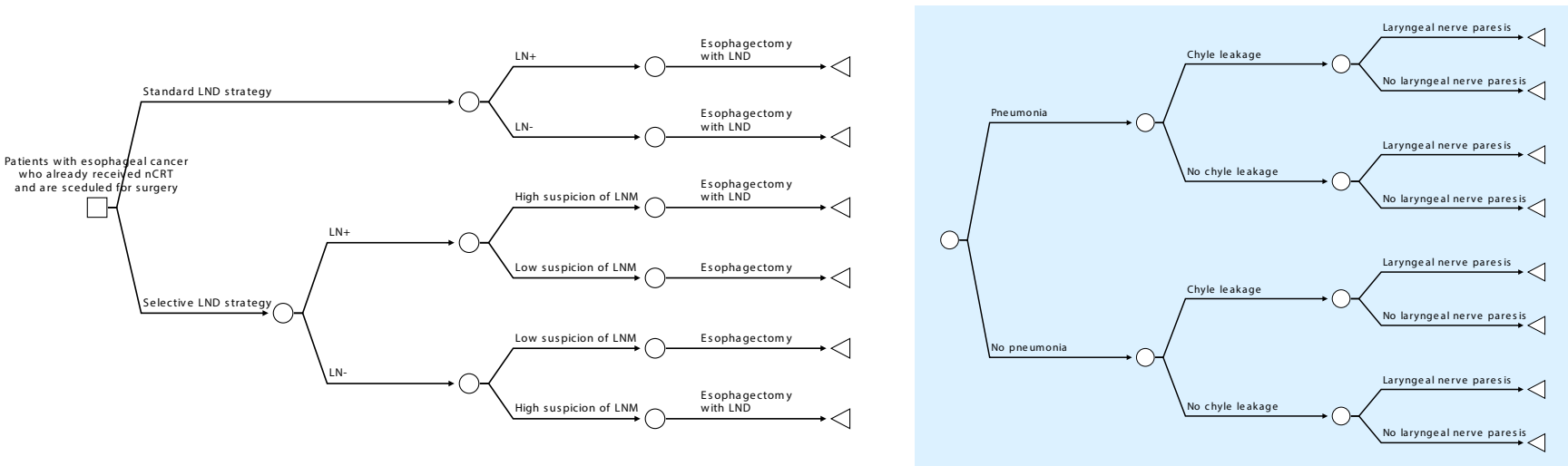

Figure 1 Decision tree of diagnosis and initial treatment. In the standard LND strategy, patients with esophageal cancer receive esophagectomy with LND regardless of their LN status. In the selective LND strategy, the restaging results after nCRT determine what treatment is given. In case of high suspicion for LNM, the patient receives esophagectomy with LND, while in case low suspicion for LN metastases, the patient receives esophagectomy. Each terminal node (triangle) is followed by the complications decision tree displayed in the blue box. LN, lymph node; LND, lymph node dissection; LNM, lymph node metastases; nCRT, neoadjuvant chemoradiotherapy. 


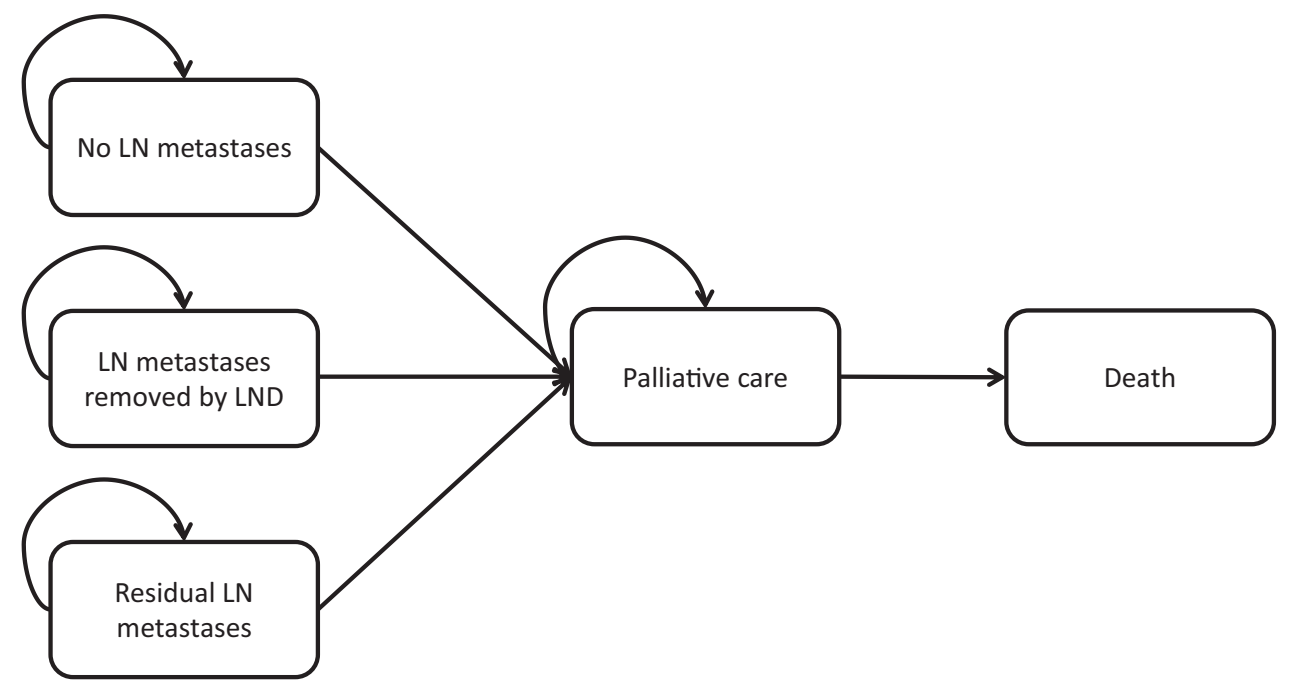

Figure 2 State-transition model of long-term follow-up. After initial treatment patients could have had no lymph node metastases and transit to the 'no LN metastases' health state. Patients with lymph node metastases could be successfully treated for their LN metastases and transit to the 'LN metastases removed by LND' health state. Patients who received no or unsuccessful treatment for their LN metastases transited to the 'residual LN metastases' health state. Patients could transit to the 'palliative care' health state in case recurrent disease was detected and from 'palliative care' to 'death'. Patients could die in every state due to non-cancer-related causes, this is not displayed. LN, lymph node; LND, lymph node dissection.

\section{State-transition model}

To simulate the long-term follow-up of patients after treatment, the decision tree was followed by a statetransition model with lifetime horizon. The health states in this model were 'no LN metastases', 'LN metastases removed by LND', 'residual LN metastases', 'palliative care', and 'death'. After the decision tree, we assumed patients without LN metastases transit to the 'no LN metastases' state with a relatively low chance of extraregional progression. ${ }^{8}$ Patients with $\mathrm{LN}$ metastases could be successfully treated for their metastases and transit to the 'LN metastases removed by LND' with the possibility of extraregional progression. ${ }^{9}$ In case restaging results falsely indicated low suspicion of LN metastases, LND was falsely omitted. Therefore, these patients transit to the 'residual LN metastases' state. We assumed these patients would receive palliative care after 6 months.

Based on biannual follow-up, a cycle time of 6 months was chosen with a lifelong time horizon. A discount rate was applied to effects, to adjust future effects to present values. A discount rate of $1.5 \%$ was applied to quality-adjusted life years (QALYs), according to Dutch guidelines. ${ }^{10}$

\section{Transition probabilities}

Transition probabilities were derived from literature or expert opinion (table 1). A probability of $31 \%$ to have $\mathrm{N}$ +disease after nCRT was used, based on van Hagen et al. ${ }^{1}$ The percentage of LNDs in which all LN metastases are successfully removed is unknown and may vary per surgeon. We therefore assumed a $94 \%$ probability of treatment success for LND (ie, 6\% of patients with residual LN metastases), which was based on the number of early locoregional recurrences after surgery with LND in van Hagen et al. ${ }^{1}$ Frequencies of LND-related complications were derived from the Dutch Upper Gastrointestinal Cancer Audit database. ${ }^{11}$ Sixty-four per cent of patients had either pulmonary complications, chyle leakage or recurrent laryngeal nerve paresis, or a combination of complications (online supplementary figure 1). The other $36 \%$ of patients had no complications or complications that would not be influenced by LND.

In the case vignette study among esophageal surgeons, experts were asked to determine the minimally required diagnostic accuracy of a new diagnostic test to omit LND, and this resulted in a median sensitivity of $92 \%$ and specificity of $90 \% .{ }^{6}$ We used these values for accuracy of the restaging results after nCRT in our model. Currently, LND is never omitted in clinical practice, therefore the percentage of complications that could be avoided when LND is omitted is unknown. In the case vignette survey, experts expected a $31 \%$ decrease in pneumonia, $72 \%$ decrease in chyle leakage, and a 57\% decrease in laryngeal nerve paresis when LND is omitted (online supplementary figure 1$){ }^{6}$

\section{Outcome measures}

Effectiveness was measured through QALYs, which is a combination of QoL (utility) and survival (table 2). A utility reflects QoL on a 0 to 1 scale, with 0 representing death and 1 representing full health. Utility scores were obtained from a cross-sectional study in esophageal cancer 
Table 1 Transition probabilities

\begin{tabular}{|c|c|c|}
\hline Parameter & Probability* & Source \\
\hline \multicolumn{3}{|l|}{ Decision tree } \\
\hline NO after nCRT & $0.689(\alpha=111 ; \beta=50)$ & van Hagen et al ${ }^{1}$ \\
\hline Treatment success rate standard LND & $0.938(\alpha=167 ; \beta=11)$ & van Hagen et al ${ }^{1}$ \\
\hline Treatment success rate of selective LND & 0.92 & de Gouw et $a l^{6}$ \\
\hline Unnecessary LND rate of selective LND & 0.10 & de Gouw et $a l^{6}$ \\
\hline Chance of pneumonia & $0.523(\alpha=1120 ; \beta=1023)$ & DUCA database ${ }^{11}$ \\
\hline Chance of chyle leakage & $0.150(\alpha=153 ; \beta=870)$ & DUCA database ${ }^{11}$ \\
\hline Chance of laryngeal nerve paresis & $0.123(\alpha=99 ; \beta=708)$ & DUCA database ${ }^{11}$ \\
\hline Chance of chyle leakage when pneumonia is present & $0.099(\alpha=111 ; \beta=1009)$ & DUCA database ${ }^{11}$ \\
\hline $\begin{array}{l}\text { Chance of laryngeal nerve paresis when pneumonia is } \\
\text { present }\end{array}$ & $0.067(\alpha=65 ; \beta=899)$ & DUCA database ${ }^{11}$ \\
\hline $\begin{array}{l}\text { Chance of laryngeal nerve paresis when chyle leakage } \\
\text { is present }\end{array}$ & $0.049(\alpha=7 ; \beta=135)$ & DUCA database ${ }^{11}$ \\
\hline $\begin{array}{l}\text { Chance of laryngeal nerve paresis when pneumonia and } \\
\text { chyle leakage are present }\end{array}$ & $0.119(\alpha=12 ; \beta=89)$ & DUCA database ${ }^{11}$ \\
\hline Relative risk of pneumonia without LND & $0.689($ IQR $0.457-1) \dagger$ & de Gouw et $a l^{6}$ \\
\hline Relative risk of chyle leakage without LND & $0.276($ IQR $0.065-0.526) \dagger$ & de Gouw et $a l^{6}$ \\
\hline Relative risk of laryngeal nerve paresis without LND & $0.431($ IQR $0.154-1) \dagger$ & de Gouw et al ${ }^{6}$ \\
\hline Remaining OR time without LND & $0.800($ IQR $0.667-0.833) \dagger$ & de Gouw et $a l^{6}$ \\
\hline \multicolumn{3}{|l|}{ State-transition model } \\
\hline Chance of recurrence in patients with NO after nCRT & $0.475(\alpha=28 ; \beta=31)$ over 7 years $\neq$ & Shapiro et $a l^{8}$ \\
\hline Chance of recurrence in patients with N1 after nCRT & $0.690(\alpha=80 ; \beta=36)$ over 7 years $\neq$ & Shapiro et $a l^{8}$ \\
\hline $\begin{array}{l}\text { Chance of palliative care in patients with residual LN } \\
\text { metastases }\end{array}$ & 1.00 & Assumption \\
\hline Standard death rate & Age dependent mortality rates & Statistics Netherlands ${ }^{18}$ \\
\hline Cancer related death ('palliative treatment' to 'death') & $0.930(\alpha=159 ; \beta=12)$ over 2 years $\neq$ & Parry et $a l^{19}$ \\
\hline
\end{tabular}

${ }^{*} \beta$-distributions were assigned to the parameters for use in the probabilistic sensitivity analysis. The characteristics of the $\beta$-distribution are presented between brackets, as an $\alpha$ and $\beta$ value (where $\alpha$ represents the number of events in a sample and $\beta$ the number of non-events). †Data was sampled from the original study data.

$\ddagger$ Probabilities were converted to bi-annual probabilities using the formulas: $r=-\log (1-p)$ and $p=1-e^{(-r t)} .20$

DUCA, Dutch Upper Gastrointestinal Cancer Audit; LN, lymph node; LND, lymph node dissection; N0, no lymph node metastases; N1, presence of lymph node metastases; nCRT, neoadjuvant chemotherapy; OR, operating room.

patients which measured EuroQol-5D utilities during surveillance, progressive disease and palliative care. ${ }^{12}$ Utility scores for the first 6 months after surgery were calculated from a multicenter randomized controlled trial that investigated open versus minimally invasive esophagectomy. ${ }^{13}$ We calculated the utility for having no or LND-unrelated complications and disutilities for developing a pneumonia, chyle leakage or recurrent laryngeal nerve paresis. We assumed that the disutility for developing a complication would influence QoL in the first 6 months. ShortForm-36 values from Biere $e t$ al were converted to EuroQol-5D utilities by the algorithm of Ara et al. ${ }^{1314}$

\section{Analysis}

A hypothetical cohort of patients was sent through the model to evaluate short-term and long-term consequences.
Short-term consequences of both strategies included percentages of patients that received LND, number of complications and the percentage of early locoregional recurrences. Long-term consequences included QALYs and survival.

\section{Threshold analyses}

We assumed that using the restaging results after nCRT to select patients for LND would result in $92 \%$ treatment success, that is, no recurrence due to residual LN metastases, and in $10 \%$ unnecessary LNDs, that is, LND performed while no LN metastases were present, based on the expert case vignette survey. However, using the restaging results could potentially have different results. We determined which combinations of treatment success and unnecessary LNDs resulted in equal QoL (ie, QALYs) for both strategies. 
Table 2 Utilities

\begin{tabular}{|c|c|c|}
\hline Parameter & Utility* & Source \\
\hline Disutility for experiencing a pneumonia & 0.079 & Biere et $a^{13}$ \\
\hline Disutility for experiencing a chyle leakage $†$ & 0 & Biere et $a l^{13}$ \\
\hline Utility in the first 6 months in the no evidence of disease state & $0.729(\mathrm{SD}=0.259 ; \mathrm{n}=32)$ & Biere et $a l^{13}$ \\
\hline Utility after the first 6 months in the no evidence of disease state & $0.800(\mathrm{SD}=0.21 ; \mathrm{n}=75)$ & Doherty et $\mathrm{al}^{12}$ \\
\hline Utility in the palliative care state & $0.740(\mathrm{SD}=0.19 ; \mathrm{n}=26)$ & Doherty et al ${ }^{12}$ \\
\hline
\end{tabular}

${ }^{\star} \beta$-distributions were assigned to the parameters for use in the probabilistic sensitivity analysis. The characteristics of the $\beta$-distribution are presented between brackets, as an SD and $\mathrm{n}$.

†Patients with chyle leakage and laryngeal nerve paresis had a higher utility than patients without complications, therefore the disutility was zero.

.LN, lymph node.

Routine LND practice varied considerably in extent and in the specific LN stations that are dissected. It is therefore likely that this influences the treatment success of LND. In the base case analysis, we assumed treatment success in $94 \%$ of patients (ie, $6 \%$ residual LN metastases). In case treatment success of LND is higher or lower than expected, the effectiveness of both strategies will change. We determined at which point both strategies result in equal QoL.

\section{Sensitivity analysis}

We determined the influence of uncertainty in treatment success of LND, the percentage of patients with $\mathrm{N} 0$ after nCRT, and the disutilities assigned to complications on QoL outcomes. Disutilities were varied from 0 (0 days of full health lost) to 0.5 (183 days of full health lost), treatment success was varied from $80 \%$ to $100 \%$ and N0 after nCRT was varied from $50 \%$ to $95 \%$. The results are displayed in a tornado diagram.

To investigate sampling uncertainty concerning the different parameters in the model, a probabilistic sensitivity analysis with 10,000 simulations was performed. Distributions were estimated for all uncertain parameters in the model, except for assumptions, characteristics of the new test and disutilities which were incorporated in threshold analyses and tornado diagrams. Parameters based on the worldwide case vignette survey were sampled from the original data and 95\% CIs were calculated using the percentile method and displayed between brackets.

\section{RESULTS}

Using a selective LND approach based on restaging results after nCRT, with an assumed treatment success rate of $92 \%$ and $10 \%$ unnecessary LNDs, could potentially result in a $65 \%$ (59\% to $70 \%$ ) decrease in LNDs. Due to the decrease in number of performed LNDs, a $10.6 \%, 4.9 \%$ and $2.8 \%$ decrease in pneumonias, chyle leakages and in recurrent laryngeal nerve paresis might be accomplished (table 3). On the other hand, there seems a slight increase in patients with residual LN metastases of $0.6 \%(-0.7 \%$ to $1.6 \%)$, which has a huge impact on the quality of life of these patients. Although uncertain, the long-term effectiveness in QALYs seems lower, as the decrease in complications appears not to outweigh

Table 3 Outcomes

Standard LND strategy Selective LND strategy Increment

\begin{tabular}{lccc}
\hline Short-term & & & \\
Percentage of LNDs $(95 \% \mathrm{Cl})$ & $100(100$ to 100$)$ & $35.4(29.7$ to 41.5$)$ & $-64.6(-70.3$ to -58.5$)$ \\
\hline $\begin{array}{l}\text { Percentage of patients with residual } \mathrm{LN} \\
\text { metastases }(95 \% \mathrm{Cl})\end{array}$ & $1.9(0.9$ to 3.3$)$ & $2.5(1.9$ to 3.1$)$ & $0.6(-0.7$ to 1.6$)$ \\
\hline $\begin{array}{l}\text { Percentage of pneumonias }(95 \% \mathrm{Cl}) \\
\text { Percentage of chyle leakages }(95 \% \mathrm{Cl})\end{array}$ & $52.3(50.2$ to 54.4$)$ & $41.7(21.8$ to 53.9$)$ & $-10.6(-30.4$ to 0$)$ \\
\hline Percentage of laryngeal nerve paresis $(95 \% \mathrm{Cl})$ & $12.3(11.0$ to 13.7$)$ & $7.4(3.9$ to 13.8$)$ & $-4.9(-8.6$ to 1.1$)$ \\
\hline Long-term & $9.1(7.9$ to 10.4$)$ & $6.4(2.9$ to 10.9$)$ & $-2.8(-6.3$ to 1.5$)$ \\
\hline Survival, years $(95 \% \mathrm{Cl})$ & $8.19(6.86$ to 9.67$)$ & $8.16(6.83$ to 9.65$)$ & $-0.03(-0.08$ to 0.03$)$ \\
\hline QALYs $(95 \% \mathrm{Cl})$ & $6.44(5.35$ to 7.68$)$ & $6.42(5.34$ to 7.67$)$ & $-0.02(-0.06$ to 0.03$)$ \\
\hline
\end{tabular}

LN, lymph node; LND, lymph node dissection; QALYs, quality-adjusted life years. 
the downside of an increase in residual LN metastases, that is, the expected QALY loss was 0.02 (0.06 QALY lost to 0.03 QALY gained).

\section{Threshold analyses}

In the base case analysis, the selective LND strategy results on average in less QoL (QALYs). To be equally effective as the standard LND strategy, the selective LND strategy should have at least $94 \%$ treatment success. In other words, the treatment success rate of selective LND should be equal to the treatment success rate of standard LND, resulting in an equal amount $(1.9 \%)$ of residual LN metastases in both strategies. The influence of unnecessary LNDs is too small to influence QoL outcomes. Residual LN metastases have therefore a larger impact on QoL than short-term complications.

Conversely, a surgical strategy with $92 \%$ treatment success and $10 \%$ unnecessary LNDs will result in equal QoL in both strategies when standard LND has a treatment success of 92\%. In this case, both standard LND and selective LND treat $92 \%$ of patients in the correct way and have an equal number of patients with residual LN metastases $(2.5 \%)$, while selective LND also decreases the amount of unnecessary complications.

\section{Sensitivity analyses}

We determined the influence of our assumptions on QoL outcomes. Treatment success rate of standard LND was the main determinant, while an increase or decrease in either the percentage of patients with N0 after nCRT or severity of complications had lower impact on quality of life (figure 3).

\section{DISCUSSION}

\section{Summary of main findings}

After nCRT, only one-third of esophageal cancer patients harbor positive locoregional LN metastases.
Our hypothesis before modeling was that by selecting patients for LND, the number of unnecessary LNDs could be reduced and unnecessary morbidity could be saved. Results of our decision analytical model show that a selective LND strategy with $92 \%$ treatment success and $10 \%$ unnecessary LNDs could have short-term benefits, that is, a decrease in the number of performed LNDs and LND-related complications. However, it may also result in a slight increase in residual locoregional LN metastases, which impacts the quality of life tremendously. Although uncertain, the long-term effectiveness in QALYs of selective LND seems lower. Furthermore, the potential added value of such a new surgical strategy is highly dependent on the treatment success rate of standard LND, which is currently unknown and will vary between surgeons. Residual LN metastases have a larger impact on QoL than short-term complications, and new surgical strategies should therefore have a treatment success rate at least equal to the treatment success rate of standard LND.

\section{Strengths and limitations}

This study showed the value of decision analytical modeling prior to surgical innovation, that is, in the pre-IDEAL stage. Our hypothesis was that by selecting patients for LND, the number of unnecessary LNDs could be reduced and unnecessary morbidity could be saved. We showed that this is not necessarily the case, therefore modeling could inform the decision whether it is worthwhile to invest in research and development of this surgical innovation. Furthermore, in case research is initiated, modeling showed the circumstances under which this surgical innovation would have added value. It can therefore serve as guidance to monitor surgical innovation. Also, this study highlighted important information gaps in current clinical practice, for example, treatment success rate of standard LND, which can now be included in research.

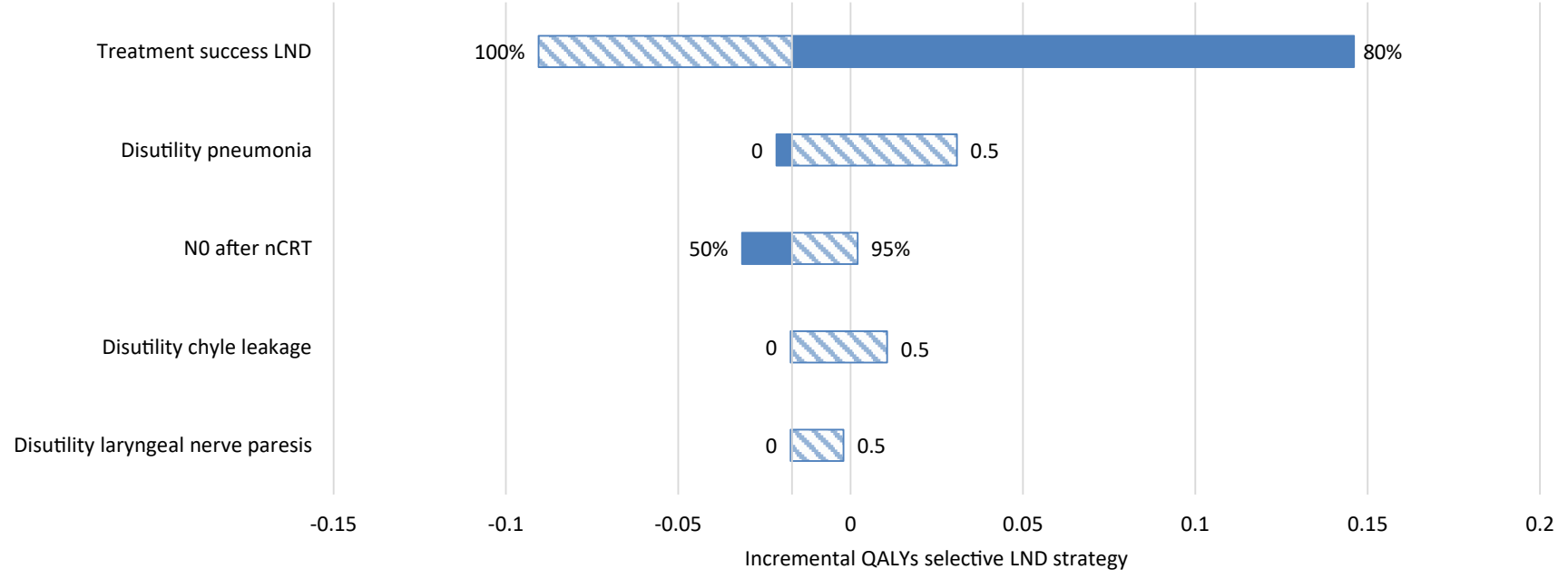

Figure 3 Tornado diagram showing the incremental QoL (QALYs) for different treatment success rates of LND, percentage of patients with N0 after nCRT, and complication-related disutilities. Value ranges are indicated in the chart. A disutility of 0.5 equals 183 days in full health that are lost. LND, lymph node dissection; nCRT, neoadjuvantchemoradiotherapy; N0, no lymph node metastases;QALYs, quality-adjusted life years; QoL,quality of life. 
Some potential limitations should also be mentioned. First, we had to make assumptions about the consequences of omitting LND, as LND is currently never omitted in esophageal cancer surgery. To inform these assumptions, we conducted a case vignette study which provided estimates of the consequences of omitting LND determined by 89 esophageal surgeons. ${ }^{6}$

Second, there was little evidence about utility values for specific complications. We were able to use real world data to construct these utilities, however this resulted in small patient groups for specific complications. ${ }^{13}$ The utility values are therefore uncertain and should be researched in more detail in future studies.

Third, it is important to note that we assumed that in the selective LND strategy, all LN metastases were always included in the LND. Although preoperative localization of LN metastases might improve results of LND, treatment success of LND might still be lower than $100 \%$ as LND success rates are highly dependent on local operative protocols. ${ }^{615}$ Moreover, it is unknown if complete LND, including all metastatic lymph nodes, leads to better survival.

Fourth, some probabilities are based on Dutch healthcare data and may therefore slightly differ from other countries. However, we expect small differences but similar trends in effectiveness. Given the detailed presentation of the model, those interested can assess the transferability of the results to their specific situation/country.

\section{Implications for clinical practice and further research}

Our results show that the consequences of having residual LN metastases are far more serious than those of short-term complications. Only when the number of patients with residual LN metastases is equal or lower than with standard LND, a new surgical strategy that selects patients for LND based on restaging results after nCRT will be more effective than standard LND. However, standard LND practice varies considerably in extent and in the specific LN stations that are dissected. ${ }^{6}{ }^{15}$ Therefore, the treatment success rate of standard LND will likely vary per surgeon, hospital and country. Since the treatment success rate is the most important determinant for the effectiveness of selective LND strategies, this should be researched in more detail to determine the real value of developing and implementing new surgical strategies. In case the treatment success rate of standard LND is lower than assumed, positive outcomes for selective LND strategies seem more likely. Once positive outcomes seem likely, other consequences of a new surgical strategy, such as its price, potential cost savings and logistic issues, should also be researched during development.

In this study we compared standard esophagectomy with standard LND to a new strategy in which LND was omitted in patients with low suspicion of LN metastases after nCRT. As esophagectomy is still performed while LND is omitted, only a limited amount of morbidity could be prevented. To increase the effectiveness of a selective LND strategy, less invasive surgical techniques such as local endoscopic resection might be an alternative approach. ${ }^{16}$ Furthermore, active surveillance for patients with a complete response (no evidence of the primary tumor or metastases after nCRT) is currently studied in two phase 3 trials. ${ }^{17}$ The already completed preSANO trial showed favorable outcomes for this treatment strategy. ${ }^{9}$ This shows that the treatment of esophageal cancer is transforming towards more personalized treatment strategies with an increasing need for personalized surgical strategies. Our model provides important guidance in the development of these strategies, and can easily be updated to assess the added value of promising surgical strategies or specific techniques for the detection of LN metastases.

\section{CONCLUSIONS}

Decision analytical modeling showed that the reduction in LND-related complications that is accomplished by selecting patients for LND seems not to outweigh a QALY loss in the long-term due to residual LN metastases. Despite the shortterm advantages of selective LND based on restaging results after nCRT, this strategy can only match long-term QALYs of standard LND when its success rate equals the success rate of standard LND.

Twitter Mirre Scholte @MirreScholte and Maroeska M Rovers @MaroeskaRovers

Acknowledgements We would like to thank the authors for the TIME trial (Biere et a) for providing the original study data. Furthermore, we would like to thank the lolaHESG community for their valuable comments on draft versions of the manuscript.

Contributors MMR, CR, DJJMdG, JG, BRK and MS conceived and designed the study. BRK and CR critically reviewed the outcomes from a clinical point of view. DJJMdG and MS acquired the data and performed the analyses with supervision of JG and MMR. DJJMdG and MS drafted the manuscript. All authors critically reviewed the manuscript and approved the final submitted version.

Funding The authors have not declared a specific grant for this research from any funding agency in the public, commercial or not-for-profit sectors.

Competing interests None declared.

Patient consent for publication Not required.

Ethics approval Ethics approval was not required as the model makes use of a hypothetical cohort of patients.

Provenance and peer review Not commissioned; externally peer reviewed.

Data availability statement All data relevant to the study are included in the article or uploaded as supplementary information. The authors confirm that the data supporting the findings of this study are available within the article and its supplementary materials. The model is available from the corresponding author, MS, upon reasonable request.

Open access This is an open access article distributed in accordance with the Creative Commons Attribution Non Commercial (CC BY-NC 4.0) license, which permits others to distribute, remix, adapt, build upon this work non-commercially, and license their derivative works on different terms, provided the original work is properly cited, appropriate credit is given, any changes made indicated, and the use is non-commercial. See: http://creativecommons.org/licenses/by-nc/4.0/.

\section{ORCID iDs}

Mirre Scholte http://orcid.org/0000-0003-3102-6011

Didi JJM de Gouw http://orcid.org/0000-0002-1414-5935

\section{REFERENCES}

1 van Hagen P, Hulshof MCCM, van Lanschot JJB, et al. Preoperative chemoradiotherapy for esophageal or junctional cancer. $N$ Engl J Med 2012;366:2074-84.

2 Little AG, Lerut AE, Harpole DH, et al. The Society of thoracic surgeons practice guidelines on the role of multimodality treatment 
for cancer of the esophagus and gastroesophageal junction. Ann Thorac Surg 2014;98:1880-5.

3 Network NCC. NCCN guidelines: esophageal and esophagogastric junction cancers. 2016.

4 Agarwal B, Swisher S, Ajani J, et al. Endoscopic ultrasound after preoperative chemoradiation can help identify patients who benefit maximally after surgical esophageal resection. Am J Gastroenterol 2004;99:1258-66.

5 Heneghan HM, Donohoe C, Elliot J, et al. Can CT-PET and endoscopic assessment Post-Neoadjuvant chemoradiotherapy predict residual disease in esophageal cancer? Ann Surg 2016;264:831-8.

6 de Gouw D, Scholte M, Gisbertz SS, et al. Extent and consequences of lymphadenectomy in esophageal cancer surgery: case vignette survey. BMJ Surgery, Interventions \& Health Technologies 2020;0:e000026.

7 Vemer P, Corro Ramos I, van Voorn GAK, et al. AdViSHE: a Validation-Assessment tool of Health-Economic models for decision makers and model users. Pharmacoeconomics 2016;34:349-61.

8 Shapiro J, van Lanschot JJB, Hulshof MCCM, et al. Neoadjuvant chemoradiotherapy plus surgery versus surgery alone for oesophageal or junctional cancer (cross): long-term results of a randomised controlled trial. Lancet Oncol 2015;16:1090-8.

9 Noordman BJ, Spaander MCW, Valkema R, et al. Detection of residual disease after neoadjuvant chemoradiotherapy for oesophageal cancer (preSANO): a prospective multicentre, diagnostic cohort study. Lancet Oncol 2018;19:965-74.

10 Zorginstituut Nederland. Richtlijn voor Het uitvoeren van economische evaluaties in de gezondheidszorg.

11 Dutch Upper GI Cancer Audit (DUCA). Annual report 2012017.
12 Doherty MK, Leung Y, Su J, et al. Health utility scores from EQ$5 \mathrm{D}$ and health-related quality of life in patients with esophageal cancer: a real-world cross-sectional study. Dis Esophagus 2018;31. doi:10.1093/dote/doy058. [Epub ahead of print: 01 Dec 2018].

13 Biere SSAY, van Berge Henegouwen MI, Maas KW, et al. Minimally invasive versus open oesophagectomy for patients with oesophageal cancer: a multicentre, open-label, randomised controlled trial. Lancet 2012;379:1887-92.

14 Ara R, Brazier J. Deriving an algorithm to convert the eight mean SF-36 dimension scores into a mean EQ-5D preference-based score from published studies (where patient level data are not available). Value Health 2008;11:1131-43.

15 Hagens ERC, van Berge Henegouwen MI, Cuesta MA, et al. The extent of lymphadenectomy in esophageal resection for cancer should be standardized. J Thorac Dis 2017;9:S713-23.

16 Ning B, Abdelfatah MM, Othman MO. Endoscopic submucosal dissection and endoscopic mucosal resection for early stage esophageal cancer. Ann Cardiothorac Surg 2017;6:88-98.

17 Noordman BJ, Wijnhoven BPL, Lagarde SM, et al. Neoadjuvant chemoradiotherapy plus surgery versus active surveillance for oesophageal cancer: a stepped-wedge cluster randomised trial. BMC Cancer 2018;18:142.

18 Statistics Netherlands. Life expectancy, 2017. Available: http:// statline.cbs.nl/Statweb/publication/?DM=SLNL\&PA=37360ned\&D1= 0\&D2=1\&D3=50-99\&D4=|\&HDR=G1, T\&STB=G2, G3\&VW =T

19 Parry K, Visser E, van Rossum PSN, et al. Prognosis and treatment after diagnosis of recurrent esophageal carcinoma following esophagectomy with curative intent. Ann Surg Oncol 2015;22 Suppl 3:S1292-300.

20 Briggs AC, K.; Sculpher M. Decision modelling for health economic evaluation. Oxford University Press, 2006. 